Pál Uzonyi

Budapest

\title{
Zur höchsten Zahl der Aktanten von verbalen Valenzträgern
}

DOI: 10.14232/fest.bassola.15

\begin{abstract}
In der Studie wird zuerst der Frage nachgegangen, ob die Termini Aktant, Ergänzung und Komplement in jedem Fall koreferent sind. Argument wird nur selten als deren Synonym verwendet, zumeist wird es auf der tieferen Ebene der Prädikat-Argument-Strukturen verortet. Stelligkeit wird vornehmlich auf Prädikate, Wertigkeit auf Valenzträger, insbesondere Verben bezogen. Wenn eine Valenzdimension exklusiv angewendet wird, z.B. Subklassenspezifik, kann die Zahl der Leerstellen 7 oder mehr erreichen, während eine Kombination von Obligatorik, Impliziertheit und Subklassenspezifik ein Maximum von 5 Mitspielern zulässt. Die Einbeziehung von Kasusrektion als Valenzdimension kann die Wertigkeit erhöhen, wenn sie der Impliziertheit übergeordnet wird.
\end{abstract}

\section{Aktanten, Ergänzungen, Komplemente (und Argumente?) - sind sie synonyme Termini?}

Die Antwort ist ein Jein, wie es auch bei ähnlichen terminologischen Fragen gewöhnlich der Fall ist. Obwohl Autoren valenzlinguistischer Werke meist konsequent nur einen dieser Termini gebrauchen (z.B. Helbig, Ágel - Aktant; Schumacher, Engel - Ergänzung; Zifonun et al. 1997 - Komplement), meinen damit alle dasselbe: ,Valenzpartner. Trotz dieses gemeinsamen Nenners kann man natürlich nicht von einer gemeinsamen Extension sprechen, wobei aber die Referenzunterschiede nicht mit der Terminuswahl, sondern mit den abweichenden Valenzbegriffen und -kriterien einhergehen, die die einzelnen Linguisten verwenden: Helbig - Notwendigkeit in einem „Stellenplan“ des Valenzträgers (z.B. in Helbig / Schenkel 1969: 33); Engel - Subklassenspezifik oder/und Obligatorik (z.B. Engel 1994: 98f.); die von Zifonun et al. (1997: 1043ff.) verwendeten Tests prüfen zur Unterscheidung der Komplemente von Supplementen die 
Valenzkriterien Obligatorik (Reduktionstest), Impliziertheit (Folgerungstest) und Subklassenspezifik (Anschlusstest). Das unscharfe Verhältnis der Termini zeigt sich auch daran, dass Schumacher (1978) bzw. Schumacher et al. (2004) mit derselben Testreihe (terminologisch) nicht Komplemente, sondern Ergänzungen erhalten. Im E-VALBU wiederum, das als eine elektronische Derivation von Schumacher et al. (2004) im Rahmen des IdS-Informationssystems grammis (https://grammis.ids-mannheim.de/verbvalenz, eingesehen am 08.01.2019) gilt, wird der Terminus Ergänzung durch Komplement ersetzt. Aktant ist letzten Endes eine Entlehnung des französischen Terminus actant von Tesnière (1959), der allerdings in der Übersetzung des Opus durch Engel (Tesnière 1980) als Ergänzung erscheint (zu weiteren Einzelheiten der Übersetzung s. Engel 1996).

Diejenigen, die in einem Werk mehrere der Termini gebrauchen, verbinden damit gemeinhin verschiedene Bedeutungen, d.h., sie behandeln sie nicht als Synonyme. Eisenberg (1994) gebraucht beispielsweise sowohl Aktant als auch Ergänzung, letzteres als zusammenfassende Bezeichnung für Subjekt, Objekt und Prädikativ, was aber bei der Erörterung valenzgebundener Adverbiale zu einigen terminologischen Schwierigkeiten führt. Eine der problematischen Fragen ist, ob obligatorische, aber nicht regierte Präpositionalgruppen Objekte sind. Da sie auf S. 296 als Ergänzungen eingestuft werden, sind sie laut folgender Aussage auf S. 90 zwangsläufig auch Objekte: „Unter den Ergänzungen eines Verbs oder prädikativen Adjektivs kann eine in der Regel als Subjekt ausgemacht werden. Alle übrigen werden Objekte genannt.“ Auch aus der Frage auf Seite 296 folgt, dass ein Adverbial keine Ergänzung sein kann: „Gibt es etwa bei einer lokalen oder temporalen, weglaßbaren PrGr [...] die Möglichkeit zu entscheiden, ob sie Ergänzung [...] oder Adverbial sind?" Generell scheint es in Richtung eines ungewöhnlichen Parallelismus zu gehen: Was Ergänzung ist, ist Subjekt oder Objekt, was Angabe ist, ist Adverbial. Helbig / Buscha (1972) vermeiden diese unerwünschte terminologische Synonymie dadurch, dass bei der Abgrenzung der Objekte gegen Adverbiale Kommutierbarkeit und Anapher (Leitwort) als grundsätzliche Kriterien verwendet werden, während bei der Differenzierung zwischen Aktanten (= Ergänzungen) und Angaben die anders zu testenden Valenzbindungen im Vordergrund stehen. Engel (1992) vereinigt die zwei Gattungen dadurch, dass er die traditionelle Einteilung in Objekt und Adverbial außer Acht lässt und zugleich die Klassifizierung durch Leitwörter behält - aber erst nach der primären Zweiteilung in Ergänzungen 
und Angaben. So kommt es zur Koexistenz von Situativergänzung vs. Lokalangabe, modifikativer Ergänzung vs. Modalangabe usw. anstelle der traditionellen Bezeichnungen Lokaladverbial, Modaladverbial usw.

Eisenberg (1994) gebraucht also wie o.a. auch den Terminus Aktant, aber in einem anderen Sinne als Ergänzung in derselben Grammatik. Aktant soll demnach ein logisch-semantischer Partizipant des Geschehens mit einer semantischen Rolle sein (vgl. „Thema als Aktant“ auf S. 118) und steht damit dem Begriff des logischen Arguments nahe. Er kommt ebd. auch in Komposita wie Subjektaktant, Objektaktant bzw. Aktantenfunktion vor.

Die Verwendung von Aktant und Ergänzung in verschiedenen Bedeutungen findet sich auch im Duden (2005). Gallmann, der Verfasser des Kapitels „Der Satz", gebraucht die Termini teilweise ähnlich wie die Grammatiken von Eisenberg (vgl. z.B. Duden 2005: 703), aber die „freien Aktanten“, die als solche zugleich Angaben sind (vgl. S. 791f.), sind mit dem Kriterium der Argumenthaftigkeit z.T. nicht vereinbar. Der passivische Agensanschluss mit von entspricht einem Argument des betreffenden Verbs (wenn es im Aktiv und Passiv als dasselbe Verb angesehen wird), aber die freien Dative mit den Rollen Benefaktiv und Malefaktiv sind nicht impliziert, also keine Argumente i.e.S., wenn man den Argumentbegriff auf subklassenspezifische Impliziertheit einschränkt.

Wie auch dem Titel „Modern“: Argumentstruktur, „traditionell“: Valenz von Steinitz (1992) zu entnehmen ist, gibt es Linguisten, für die Argument eine valenzgebundene Einheit im weiteren Sinne von Valenz bezeichnet. Diese Verwendung des Terminus ist vor allem für die Generative Grammatik (in der allerdings die Termini Valenz, Aktant, Angabe usw. spärlich gebraucht werden) und mit ihr verwandte Ansätze charakteristisch, und zwar nicht nur auf der Ebene der logischen Valenz, auf der auch „traditionelle“ Valenzgrammatiker das Wort Argument benutzen (z.B. Helbig 1992: 13ff.), sondern auch im Sinne der (oberflächen)syntaktischen Ergänzung (z.B. Dik 1978 in seiner Functional Grammar, vgl. dazu Dürscheid 2007: 205, 233).

Das Ergänzungskriterium Argumenthaftigkeit setzt offensichtlich die klare Distinktion einer syntaktischen von einer tieferen, logischen Ebene voraus. Dazwischen setzt Helbig (1992) die Ebene der semantischen Rollen, die er früher (1977) mit den Argumenten „stillschweigend weitgehend gleichgesetzt“ habe, wie er selbst anmerkt (1992: 14). 


\section{Stelligkeit von Prädikaten}

Die Prädikatenlogik, in der früher als in der Linguistik vom Zusammenhang zwischen Prädikaten (ursprünglich: Funktionen) und ihren Argumenten gesprochen wurde, begrenzt die potenzielle Zahl der Argumente nicht (vgl. z.B. Frege 1879). Logiker differenzieren oft nicht einmal zwischen den Satzgliedern, welche die vom Prädikat eröffneten Leerstellen besetzen, und den freien Satzgliedern, die die Situation beschreiben. So kann ein Satz, der laut Valenztheoretikern außer dem Verb drei Ergänzungen und zwei Angaben enthält, von Logikern als „fünfstelliges Prädikat“ behandelt werden (z.B. Davidson 1985: 158, zitiert nach Welke 2011: 89).

Auch in der Sprachwissenschaft wird die Aufnahme eines "Situationsarguments“ oder „referenziellen Arguments“ als Argument des Satzes diskutiert (vgl. Welke 2011: 90). Für diese Satzteile wird freilich das Kriterium der Argumenthaftigkeit im valenztheoretischen Sinne kaum gelten.

Eisenberg (1999: 78) unterscheidet zwischen „logischem Argument“ und „Verbargument“. Die zur Beschreibung von Verbbedeutungen verwendeten 5 Basisprädikate (vgl. Dowty 1991) verfügen über max. 2 logische Argumente (Eisenberg 1999: 77), aber die Verben, bei denen die Kasuszuweisung mithilfe der Basisprädikate und Basisrollen sinnvoll erklärt werden kann, können auch dreistellig sein (Eisenberg 1999: 79), wobei die Stelligkeit hier eher auf die Komplementzahl bezogen ist. Das Maximum der Stelligkeit im Deutschen im letzteren Sinne wird übrigens von Eisenberg (1999: 58) als 4 bestimmt. Ähnlich wie in Eisenberg (1994) können Ergänzungen auch hier keine Adverbiale sein, jedoch durchaus Präpositionalobjekte, was für Tesnières „actants“ noch nicht galt. Daher erschien ihm die Dreistelligkeit zwangsläufig als Maximum (Subjekt, direktes Objekt, indirektes Objekt, das aber keine Präposition haben konnte). Deshalb bleiben Oberflächenentsprechungen gewisser Argumente zwangsläufig außerhalb des Skopus der Tesnière'schen Valenzauffassung. Das kann sogar für Basisprädikate gelten, beispielsweise für Posses(x,y), wenn anstelle von besitzen (Eisenberg 1999: 78) das Verb verfügen gewählt wird, bei dem das Akkusativobjekt („Zweitaktant“) durch über + Akkusativ ersetzt wird. Dass Argumente auch als Präpositionalobjekte realisiert werden können, lässt sich am einfachsten mithilfe von Verben beweisen, die einen reinen und einen präpositionalen Kasus als synonyme Satelliten haben können, z.B. jm oder an jn 
schreiben, jn etwas oder nach etwas fragen usw. Wenn man obendrein Sprachen einbezieht, die nicht zu den frequentierten indoeuropäischen Sprachen der Valenztheorie (Deutsch, Französisch, Englisch) gehören und in denen die Zahl der reinen Kasus nicht auf 4 oder den Dreier „Subjekt, direktes Objekt, indirektes Objekt" begrenzt wird, z.B. Ungarisch mit (ca.) 16 reinen Kasus, muss die Stelligkeit unter anderen Aspekten durchdacht werden. Mindestens die Hälfte der ungarischen Kasus ohne Adpositionen kommen auch als semantisch unmotivierte Kasusrektion vor, z.B. számol vmivel (Kasus Instrumental) - ,rechnen mit etw.. Diese sind also keine reinen „semantischen Kasus“ wie z.B. der Terminativ (Suffix -ig ,bis'). Ebenso wie die Mehrheit der ungarischen Suffixe haben auch die deutschen Präfixe über die Rektionsfunktion hinaus i.d.R. auch adverbiale Bedeutungen, z.B. mit weiteren Schwierigkeiten rechnen (Präpositionalobjekt) vs. mit einem Taschenrechner rechnen (Instrumentalbestimmung).

Eisenberg bezieht Adverbialbestimmungen nicht in die Errechnung der Stelligkeit mit ein, wobei die semantische Rolle „Dir“ durchaus als Richtungsadverbial realisiert werden kann. Eisenberg (1999: 84) nennt die Präpositionalphrase auf den Tisch im Satz Karl stell die Vase auf den Tisch nicht Komplement, sondern Adjunkt. Wenn jedoch eine Phrase einem logischen Argument entspricht, müsste sie qua Argumenthaftigkeit Komplement sein. Gerade bei Präpositionalphrasen soll es aber anders sein, die Präposition sei nämlich nicht einmal in Präpositionalobjekten Teil der argumentbezogenen Oberflächenkonstruktion, denn nur die NP entspreche der semantischen Rolle und die Präposition drücke im obigen Satz eine Relation zwischen einem Gegenstand und einem Ort aus (Eisenberg 1999: 83). Daraus folgt, dass nur das Nominal, das den Ort bezeichnet, einem Argument entspricht. Ein Problem stellen allerdings Adverbiale dar, die keine NP enthalten, z.B. dahin, dorthin, irgendwohin. Wenn dieses obligatorisch ist, könnte man dafür die kuriose Bezeichnung „obligatorische Angabe“ (vgl. Welke 1988: 88) vorschlagen, da zu einer Angabe kein Argument gehört. Dieser Terminus passt aber nicht mehr zu fakultativen Richtungsbestimmungen wie z.B. in Ich fahre mit dem Bus (dorthin).

Zifonun et al. (1997: 694) erachten in präpositionalen Komplementen (im Sinne von Objekten) ebenso nur die NP als Pendant des Arguments, wie dem folgenden Beispielsatz zu entnehmen ist, in dem die Argumente in eckigen Klammern dargestellt sind: „[Die Landschaft] erinnerte [ihn] an [seine Heimat]" (nur seine Heimat steht in Klammern, an nicht). Im Falle der Adverbia- 
le jedoch, denen „halbautonome Argumente“ entsprechen, scheinen auch die Präpositionen Konstituenten der Oberflächenrealisierungen von Argumenten zu sein (Zifonun et al. 1997: 758ff.). Auf Seite 684 wird ebd. darauf aufmerksam gemacht, dass die Stelligkeit von Prädikaten nicht mit der Wertigkeit von Valenzträgern verwechselt werden darf. Nichtsdestoweniger soll die Zahl der Komplemente einer Verbvariante zumeist mit der der Argumente des entsprechenden Prädikats übereinstimmen (Ausnahmen sind u.a. die Kopulaverben). Eine „Aufstockung“ der Argumentzahl ist u.a. durch lassen möglich, sonst soll die höchste Zahl 3 sein. Laut Zifonun et al. (1997: 725) „gibt es im Deutschen mindestens eine genuine vierstellige Beziehung“, und zwar im Falle des Verbs sich einigen. Vier- und mehrstellige Prädikate sollen sonst durch „Aufstockung“ entstehen. In grammis (https:/grammis.ids-mannheim.de/systematischegrammatik/2133, eingesehen am 08.01.2019) wird bereits wie folgt formuliert: „Neben den durch ,Aufstockung entstandenen vier- und mehrstelligen Beziehungen finden sich im Deutschen auch vier [sic!] und fünfstellige Beziehungen“. Das ist auch deshalb eine besser nachvollziehbare Formulierung, weil VALBU (Schumacher et al. 2004) - zwischendurch als E-VALBU Teil von grammis -, das 7 Jahre später als die IdS-Grammatik (Zifonun et al. 1997) erschienen ist, 16 vierstellige Verbvarianten verzeichnet, die keine Adverbialkomplemente enthalten. Falls auch Adverbialkomplemente (etwa als halbautonome Argumente) zugelassen werden, dann kommen weitere 81 vierstellige Verbvarianten hinzu, plus 3 fünfstellige ohne Aufstockung: mieten, vermieten, sich treffen (1).

\section{Stelligkeit vs. Wertigkeit von Verben}

Auch wenn die Zahl der Argumente und Komplemente bei Prädikaten und entsprechenden Verbbedeutungen zumeist gleich sein soll (Zifonun et al. 1997: 684), gibt es einen Umstand, der zu bedeutenden Unterschieden führen kann: Zur Differenzierung zwischen den Argumenten von Prädikaten und den Modifikatoren (Pendants der Angaben/Supplemente) ist bisher kein Instrumentarium ausgearbeitet worden, das mit den vielen praxisorientierten Oberflächentests zur Abgrenzung der Ergänzungen/Komplemente von den Angaben/ Supplementen in der Valenzlinguistik vergleichbar wäre. Aus dieser Vielzahl ist es am ehesten der Folgerungstest (Implikationstest), der einem engen Ar- 
gumentbegriff nahe kommt. Ergänzt durch den Anschlusstest schließt der Folgerungstest „Situationsargumente" (z.B. nicht-subklassenspezifische Lokaladverbiale, Temporaladverbiale usw.) aus, deren Komplementstatus in den meisten Valenzansätzen nicht in Frage kommt. Verschiedene Valenztests knüpfen an differente Valenzdimensionen an (vgl. Jacobs 1994), was nicht nur mit unterschiedlichen E/A-Grenzen, sondern auch mit diversen Ergänzungszahlen (sprich: Wertigkeiten) pro Verb einhergeht.

Das Kriterium von Helbig / Buscha (1972: 549) „im Stellenplan des Verbs enthalten“ würde als etwa ,im Satzmodell des Verbs enthalten' gedeutet einen Zirkelschluss darstellen, wenn der Begriff „Besetzungen von Leerstellen“ ebd. nicht mit der eingeklammerten Paraphrase "Argumente des Prädikats“ gleichgesetzt würde. Dadurch wird aber implizite dem Kriterium der Argumenthaftigkeit eine bestimmende Rolle zugesprochen. Es ist bedauerlich, dass diese „argumenthaften“ Aktanten gerade mithilfe des als-Tests von Angaben, die „reduzierte Sätze“ darstellen, exemplarisch abgegrenzt werden, da nur eine begrenzte Gruppe der Angaben (vornehmlich Lokaladverbiale) mit dem Test richtig funktionieren. Bei temporalen, kausalen usw. Angaben ist das nicht möglich. Bereits der im Vorwort von Helbig / Schenkel (1969: 39) verwendete Terminus Notwendigkeit lässt übrigens auf Impliziertheit, Beteiligtheit schließen, die Differenzierung zwischen notwendig und nicht-notwendig sei nämlich „in der Tiefenstruktur angelegt“. Diese Art Notwendigkeit als Synonym von Valenzgebundenheit ist natürlich nicht identisch mit einer oberflächensyntaktischen Notwendigkeit (NOT bei Jacobs 1994: 14), d.h. mit der Obligatorik. Aufgrund dieser „Sinnnotwendigkeit“ ist sowohl in Helbig / Schenkel (1969) als auch in Helbig / Buscha (1972: 556f.; 2001: 523, 525) die Vierwertigkeit das Maximum (mit welchem Begriff der Notwendigkeit diese Zahl korrespondiert, soll jetzt dahingestellt bleiben).

Das Grundkriterium der Valenz ist bei Engel (1992: 200) die Subklassenspezifik, mit der er ebenfalls zu einem Maximum von 4 Ergänzungen in seinen Satzmustern kommt. Von den insgesamt 5 Satzmustern mit 4 Ergänzungen sind es zwei, die keine Adverbiale im herkömmlichen Sinne enthalten: „sub akk dat prp“ und „sub akk prp prp“. In diesem Zusammenhang ist es natürlich bei weitem nicht belanglos, was Engel unter Subklassenspezifik versteht, wie er diese definiert und in der Praxis anwendet. Wenn nur von der Definition in seiner Grammatik (1992: 183) ausgegangen wird („Ergänzungen kommen 
nur bei bestimmten Subklassen von Verben vor, [...]"), stößt man auf etliche Widersprüche (vgl. z.B. Uzonyi 2004), wobei die Zahl der Ergänzungen pro Satzmuster leichthin mit Abstand die höchste im „Wettbewerb“ mit anderen Valenzkriterien werden kann. Wenn nämlich die bei Direktivergänzungen auch von Engel verwendete semantische Subklassenspezifik (sie können ja anders als Situativergänzungen in derselben Grammatik auch fakultativ sein) auf andere semantische Klassen der Adverbiale ausgeweitet wird, müssten Zeitdauerbestimmungen (Subklasse: durative Verben), Instrumental- und Finalbestimmungen (Subklasse: agentive Verben) usw. gleichfalls einbezogen werden. So dürfte beispielsweise das Verb essen 7 oder mehr Ergänzungen dieser Art an sich binden: jd isst (etw) (igdwie lange) (mit igdwelchem Ziel) (mit igdeinem Mittel) (igdwoher) (jm als dativus sympathicus, der bei Engel Ergänzung ist) usw. (vgl. auch Uzonyi 2008).

Der sog. enge Ergänzungsbegriff von Adamzik (1992) umfasst die vom Verb „morphosyntaktisch bestimmten“ Glieder des Satzes, wozu verhältnismäßig zuverlässige Tests (z.B. Kommutationstest) herangezogen werden können. Schwierigkeiten gibt es nur mit einigen stark begrenzt oder überhaupt nicht austauschbaren Kasusformen bei bestimmten semantischen Rollen. Hierzu gehört der Dativ in der Rolle des Benefaktivs, der u.U. durch für + Akk ersetzt werden kann, aber das ist immer noch weit von der formalen Flexibilität der Adverbiale entfernt. Der Dativ des Malefaktivs (dativus incommodi) lässt nicht einmal diese Ersetzung durch eine Präpositionalphrase mit für zu. Bei diesen Dativen spricht Engel (1992: 193) aufgrund der Subklassenspezifik von einem Ergänzungsstatus, während der morphosyntaktisch ebenso feste ethische Dativ aufgrund der fehlenden Subklassenspezifik als Angabe eingestuft wird. Der Folgerungstest, der weglassbare Satzteile auf ihre Argumenthaftigkeit i.e.S. prüfen soll, wird natürlich sowohl dativus commodi als auch dativus incommodi ausfiltern, weil beide von den meisten Verben, bei denen sie vorkommen, nicht impliziert werden (man kann z.B. einen Korb tragen, ohne dass der Korb jemandem getragen würde). Sollte der Benefaktiv doch ein implizierter Beteiligter sein, z.B. im Falle von schmeicheln, ist der Dativ in der Regel nicht ersetzbar durch andere Formen, daher spricht man hier gewöhnlich nicht von einem freien Dativ. Die Rolle Malefaktiv ist anders, da eine dativische Realisierung nicht durch eine Präpositionalphrase ersetzbar ist. Deshalb ist es problematischer, den Dativ bei passieren oder geschehen eindeutig als Kasusrek- 
tion oder als freien Dativ einzustufen. Hier reicht demnach das Kriterium der morphosyntaktischen Bestimmtheit an sich nicht aus, um Ergänzungen von Angaben zu trennen. Laut Engel (1994: 157) hilft hier aber die Subklassenspezifik weiter (Subklasse, ungesteuertes Geschehen'), die für passieren ebenso gilt wie für (intransitives) zerbrechen. Das Kriterium der Argumenthaftigkeit kann demgegenüber nur dann einen Komplementstatus rechtfertigen, wenn ,etwas geschieht' und ,etwas geschieht jemandem' als zwei distinkte Verbvarianten behandelt werden. Die Leerstelle für einen Malefaktiv scheint tatsächlich oft vom Subjektreferenten abzuhängen: Die meisten Ereignisse im Weltall geschehen offenbar ohne eine betroffene Person, ein Unrecht geschieht hingegen immer jemandem, auch wenn die konkrete Person unerwähnt bleiben kann.

Dass ein reiner Dativ nicht in jedem Fall ein Dativkomplement sein muss, ist allgemein bekannt, auch wenn er immer durch ein Leitwort (Pronomen) im Dativ substituierbar ist. Beim Akkusativ geht aber herkömmlich in jedem Fall mit dem Komplementstatus einher, dass das Leitwort ein Pronomen im Akkusativ sein muss. Wenn das Leitwort hingegen ein Adverb ist, ist bei Zeitpunkten meist die Einstufung als Supplement üblich (z.B. Sie war diesen Sonntag anderswo), bei Zeitdauern aber in der Regel als Komplement (z.B. Das dauert eine Viertelstunde). Soll dies bedeuten, dass ein Akkusativ tatsächlich immer Komplement ist, wenn das Leitwort (die Anapher) ein Pronomen im Akkusativ ist? Nun, nach dem Valenzkriterium Kasusrektion müsste es so sein. Wenn jedoch das Kriterium Impliziertheit hinzukommt, muss man bei einigen Verbbedeutungen feststellen, dass die Referenz des Akkusativobjekts aus der betreffenden Verbbedeutung nicht zwangsläufig folgt. Demnach kann man z.B. nur so essen, dass man etwas isst (das Gegessene ist impliziert), aber z.B. tanzen kann man so, dass man etwas tanzt, aber auch so, dass man nichts tanzt. Oder man kann Servietten so falten, dass diese auch danach einfach als Servietten bezeichnet werden können, aber man kann sie auch so falten, dass man dabei einen Schmetterling oder eine Bischofsmütze faltet. Die beiden Akkusativobjekte haben verschiedene semantische Rollen: Serviette, Papier, Wäsche usw. falten Patiens; einen Schmetterling, Papierflieger usw. falten - Resultat. Nur das Patiens ist impliziert, das Resultat nicht. Der Referent des Patiens-Akkusativs wird dabei im Falle der Nennung eines Resultats durch aus + Dativ ausgedrückt: Aus dem Briefpapier faltete sie einen Flieger. Das Verb falten wird im DUW (2001) wie folgt definiert: „sorgfältig zusammenlegen, sodass an der umgeschlagenen 
Stelle eine Falte (1a), ein Knick entsteht: einen Brief [zweimal] falten“. Dabei wird aus dem rechteckigen Brief wahrscheinlich ein anderes Rechteck gefaltet, aber das ist kein neuer Gegenstand, der als Resultat der Handlung zu versprachlichen wäre. Wenn also die Impliziertheit als exklusives Hauptkriterium gewählt wird, können Akkusativobjekte in der Rolle Inhalt oder Resultat bei Verben wie tanzen, winken, brüllen, falten, biegen usw. wahrscheinlich keine Ergänzungen sein. Den im syntaktischen Sinne ziemlich störenden Fall, als Akkusativobjekt kein Valenzpartner zu sein, kann man einerseits dadurch vermeiden, dass man von unterschiedlichen Verbvarianten spricht (wie VALBU bei tanzen), oder dass man Kasusrektion als Valenzkriterium der Impliziertheit vorzieht (wie z.B. Uzonyi 2009).

Analog beurteilt werden können einige mit Direktivadverbialen verknüpfte Akkusativobjekte, die nicht aus der Bedeutung des Verbs folgen, z.B. den Brief vom Tisch niesen oder den Schauspieler fünfmal auf die Bühne applaudieren (vgl. die konstruktionsgrammatische Perspektive z.B. bei Smirnova / Mortelmans 2010: 163). DUW (2001) definiert niesen wie folgt: „(infolge einer Reizung der Nasenschleimhaut) die Luft ruckartig und mit einem lauten Geräusch durch Nase und Mundraum ausstoßen“. Wenn infolge dieses Vorgangs etwas irgendwoher irgendwohin kommt, ist es im Normalfall nicht vom Niesenden intendiert. Bei Papierflieger falten kann man noch u.U. mit dem Merkmal ,Intention, etwas herzustellen' für eine andere Bedeutung als die von Papier falten argumentieren, aber bei niesen spielt die Absicht keine Rolle.

Was kann man nun vor diesem Hintergrund über die Stelligkeit des Prädikats ,niesen' bzw. über die Valenz des Verbs niesen ${ }^{1}$ sagen? Nicht-implizierte Beteiligte sind keine Argumente i.e.S., weil sie nicht als kontextunabhängiger Teil des Prädikats funktionieren, sondern nur optionell hinzugefügt werden, wie es für Modifikatoren charakteristisch ist. Aber dieselben nicht-implizierten Beteiligten können im Bereich der syntaktischen Valenz als Ergänzungen/ Komplemente erscheinen, wenn die Form des Ausdrucks eine Invarianz bezüglich Kasus oder Adposition aufweist, also vom Verb regiert wird. Das kann in manchen Fällen zu einer höheren Wertigkeit (d.h. Zahl der Valenzpartner) des

Dieses Verb ist leider in keinem deutschen Valenzwörterbuch verzeichnet, nur husten kommt im VALBU mit einer ähnlichen Verbvariante vor: „husten 2: jemand/etwas hustet etwas irgendwohin“, allerdings nur im Sinne ,Absonderung, z.B. jemand hustet Blut ins Taschentuch. 
Verbs im Vergleich zur Stelligkeit (d.h. Zahl der Argumente) des entsprechenden Prädikats führen.

Ähnlich, aber keinesfalls gleich verhält es sich im Fall der formalen Subjekte und Objekte, bei denen der obligatorischen invarianten Form der Oberfläche (es) kein Referent entspricht, weswegen sie auf der Ebene der Prädikat-Argument-Struktur nicht einmal als Modifikatoren erscheinen können.

Unter den adverbialen Bedeutungsklassen kommt die Direktivergänzung (das Direktivkomplement) in diversen Valenzansätzen am häufigsten vor, weil sie nach mehreren Kriterien ein Valenzpartner ist: Sie ist subklassenspezifisch, weil sie nicht mit allen Verben kompatibel ist; sie ist auch impliziert, weil die Notwendigkeit einer Richtung aus der Bedeutung der betreffenden Verben folgt. Aber wie oben beim Verb niesen zu sehen war, kann eine Richtung auch eine situationsabhängige Option sein. Auch das Verb lügen ist weit entfernt von den Bewegungsverben, aus deren Bedeutung prototypisch eine Richtung folgt, aber sogar lügen findet man in entsprechenden Situationen mit Richtungsbestimmungen, z.B. ins Mikrophon lügen, in die Kamera lügen (sie sind nicht in dem Maße idiomatisiert wie ins Gesicht oder in die Tasche lügen). Aufgrund der Subklassenspezifik wären diese Richtungsbestimmungen Ergänzungen, aber sie folgen nicht aus der Bedeutung von lügen, anders als ein Adressat (jm gegenüber) oder der Inhalt der Lüge (etwas), wobei die Realisierung dieser implizierten Beteiligten eine ähnlich niedrige Vorkommenshäufigkeit aufweist wie die der besagten Richtungsadverbiale (vgl. Uzonyi 2009). Völlig blockiert sind sie also nicht, was den Ausführungen von Steinitz (1992) teilweise widerspricht. Belege finden sich in Korpora, die Realisierungsmöglichkeit eines Akkusativkomplements ist sogar lexikografisch erfasst (z.B. DUW, VALBU). Anders sieht es jedoch bei den Verben belügen und anlügen aus, denen dieselbe Denotation (sprich: dasselbe Prädikat mit denselben Argumenten) entspricht wie dem Verb lügen. Die Stelligkeit ist also dieselbe: 3 Argumente, die Rollen sind Agens, Adressat und Inhalt. Der Adressat ist bei belügen und anlügen eine obligatorische Ergänzung, der Inhalt lässt sich aber nur durch komplexe Umschreibungen ausdrücken, die nach den gängigen Ergänzungsbegriffen nicht als Valenzrealisierungen eingestuft werden können, z.B. Er belog die Wähler, als er sagte, dass ...; das Gefüge eines Temporalsatzes mit einem subordinierten Objektsatz wird keinem herkömmlichen formalen Ergänzungstyp gerecht. Bei diesen Verben ist daher eine Argumentstelle auf der Ebene der syntaktischen Valenzrealisierung 
tatsächlich blockiert, woraus eine niedrigere Wertigkeit (2) resultiert als die zugrunde liegende Stelligkeit (3). Der Argumentstruktur entspricht am besten das Synonym vorlügen, bei dem jedem Argument eine obligatorische Ergänzung entspricht (vgl. Uzonyi 2009).

\section{Zusammenfassung}

Im multidimensionalen Valenzmodell spielt die Dimension der Argumenthaftigkeit eine besondere Rolle, die für viele Linguisten mit einer Zurückführung der lexikalisch-syntaktischen Valenz auf logisch-semantische Wurzeln einhergeht. Da aber auch die anderen Dimensionen in die E/A-Abgrenzung der diversen Valenzversionen einbezogen werden, erhält man oft verschiedene Zahlen für Stelligkeit (Argumente) und Wertigkeit (Ergänzungen) bei ein und derselben Verbbedeutung. Der Terminus Argument kann natürlich ebenso wie Ergänzung, Komplement oder Aktant unterschiedlich ausgelegt werden. Im vorliegenden Aufsatz wurde der Begriff auf vom Prädikat implizierte Mitspieler begrenzt, die subklassenspezifisch sind. Die diesem entsprechenden fakultativen Komplemente werden von Zifonun et al. (1997: 1043ff.) bzw. von Schumacher et al. (2004: 26f.) mithilfe eines Implikationstests und darauf folgenden Anschlusstests ausgewählt (die obligatorischen scheiden mit dem Reduktionstest in der vorangehenden Phase bereits aus). Wenn die Definition der Subklassenspezifik von Engel (1994) wörtlich genommen wird, nicht differenzierend zwischen formaler und semantischer Spezifik, können Verben leichthin 7 oder mehr „Ergänzungen“ dieser Art an sich binden (während die Satzmuster von Engel 1992 höchstens 4-wertig sind). Wenn die subklassenspezifischen Glieder Adverbiale sind, ist es nachvollziehbar, dass manche davon durch einen anschließenden Folgerungstest als Angaben ausgefiltert werden (z.B. etw. mit etw. suchen). Mit Vorbehalt spricht man hingegen von einer Angabe, wenn das nicht-implizierte Satzglied ein Objekt ist (etw. tanzen). Stelligkeit ist höher als Wertigkeit, wenn Argumentstellen syntaktisch blockiert sind (z.B. ,Inhalt ' bei belügen oder anlügen). 


\section{Literatur}

Adamzik, Kirsten (1992): Ergänzungen zu Ergänzungen und Angaben. In: Deutsche Sprache 20, 289-313.

Davidson, Donald (1985): Handlung und Ereignis. Frankfurt a.M: Suhrkamp. Dik, Simon C. (1978): Functional Grammar. Amsterdam: North-Holland Publishing Company.

Dowty, David (1991): Thematic Proto-Roles and Argument Selection. In: Language 67, No. 3, 547-619.

Duden (2005): Die Grammatik. 7., völlig neu erarb. und erw. Aufl. Mannheim / Leipzig / Wien / Zürich: Dudenverlag.

Dürscheid, Christa (2007): Syntax: Grundlagen und Theorien. 4., überarb. und erg. Aufl. Göttingen: Vandenhoeck \& Ruprecht.

DUW = Wermke, Matthias et al. (2001): Duden Deutsches Universalwörterbuch .

4., neu bearb. und erw. Aufl. Mannheim / Leipzig / Wien / Zürich: Dudenverlag. Eisenberg, Peter (1994): Grundriß der deutschen Grammatik. 3., überarb. Aufl. Stuttgart / Weimar: Metzler.

Eisenberg, Peter (1999): Grundriß der deutschen Grammatik. Bd 2: Der Satz. Stuttgart / Weimar: Metzler.

Engel, Ulrich (1992): Deutsche Grammatik. 2., verb. Aufl. Heidelberg / Budapest: Julius Groos / Múzsák.

Engel, Ulrich (1994): Syntax der deutschen Gegenwartssprache. 3., völlig neu bearb. Aufl. Berlin: Erich Schmidt (Grundlagen der Germanistik 22).

Engel, Ulrich (1996): Tesnière mißverstanden. In: Gréciano, Gertrud / Schumacher, Helmut (Hrsg.): Lucien Tesnière - Syntaxe structurale et opérations mentales. Akten des deutsch-französischen Kolloquiums anläßlich der 100. Wiederkehr seines Geburtstages, Strasbourg 1993. Tübingen: Niemeyer (Linguistische Arbeiten 348), 53-61.

Frege, Gottlob (1879): Begriffsschrift. Eine der arithmetischen nachgebildete Formelsprache des reinen Denkens. Halle a.d. S.: Louis Nebert. grammis = https:/grammis.ids-mannheim.de (eingesehen am 08.01.2019). Helbig, Gerhard (1977): Zur semantischen Charakterisierung der Argumente des Prädikats. In: Helbig, Gehard (Hrsg.): Probleme der Bedeutung und Kombinierbarkeit im Deutschen: ein Sammelband für den Fremdsprachenunterricht. Leipzig: Enzyklopädie, 40-92. 
Helbig, Gerhard (1992): Probleme der Valenz- und Kasustheorie. Tübingen: Niemeyer (Konzepte der Sprach- und Literaturwissenschaft 51).

Helbig, Gerhard / Buscha, Joachim (1972): Deutsche Grammatik. Leipzig: Enzyklopädie.

Helbig, Gerhard / Buscha, Joachim (2001): Deutsche Grammatik. Ein Handbuch für den Ausländerunterricht. Berlin / München / Wien / Zürich / New York: Langenscheidt.

Helbig, Gerhard / Schenkel, Wolfgang (1969): Wörterbuch zur Valenz und Distribution deutscher Verben. Leipzig: Bibliographisches Institut.

Jacobs, Joachim (1994): Kontra Valenz. Trier: Wissenschaftlicher Verlag (Fokus, Linguistisch-philologische Studien 12).

Schumacher, Helmut (1986): Verben in Feldern - Valenzwörterbuch zur Syntax und Semantik deutscher Verben. Berlin / New York: de Gruyter.

Smirnova, Elena / Mortelmans, Tanja (2010): Funktionale Grammatik: Konzepte und Theorien. Berlin / New York: de Gruyter.

Steinitz, Renate (1992): „Modern“: Argumentstruktur, „Traditionell“: Valenz Versuch eines Brückenschlags. In: Linguistische Berichte 137, 33-44.

Tesnière, Lucien (1959): Eléments de syntaxe structurale. Paris: Klincksieck.

Tesnière, Lucien (1980): Grundzüge der strukturalen Syntax. Hrsg. und übersetzt von Ulrich Engel. Stuttgart: Klett-Cotta.

Uzonyi, Pál (2004): Valenz kontra Subklassenspezifik. In: Czicza, Dániel / Hegedűs, Ildikó / Kappel, Péter / Németh, Attila (Hrsg.): Wertigkeiten, Geschichten und Kontraste. Festschrift für Péter Bassola zum 60. Geburtstag. Szeged: Grimm, 233-237.

Uzonyi, Pál (2008): Lexikographische Unterstützung der Übertragung von syntaktischen Strukturen. In: Jesenšek, Vida / Oštir, Alja Lipavic (Hrsg.): Wörterbuch und Übersetzung. 4. Internationales Kolloquium zur Lexikographie und Wörterbuchforschung, Universität Maribor, 20. bis 22. Oktober 2006. Hildesheim / Zürich / New York: Olms (Germanistische Linguistik 195-196), 209-220.

Uzonyi, Pál (2009): Fakten lügen nicht - Eine kontrastive Fallstudie zur Verbvalenz. In: Adamcová, Lívia / Péteri, Attila (Hrsg.): Aspekte der Fremdsprachenlinguistik in der Slowakei und in Ungarn. Bratislava / Budapest: Eötvös-Loránd-Universität / Wirtschaftsuniversität Bratislava, 185-204. 
VALBU = Schumacher, Helmut / Kubczak, Jacqueline / Schmidt, Renate / de Ruiter, Vera (2004): VALBU - Valenzwörterbuch deutscher Verben. Tübingen: Narr.

Welke, Klaus (1988): Einführung in die Valenz- und Kasustheorie. Leipzig: Bibliographisches Institut.

Welke, Klaus (2011): Valenzgrammatik des Deutschen: eine Einführung. Berlin / New York: de Gruyter.

Zifonun, Gisela / Hoffmann, Ludger / Strecker, Bruno et al. (1997): Grammatik der deutschen Sprache. 3. Bde. Berlin / New York: de Gruyter (Schriften des IDS 7). 